\title{
La mujer rural, las comadronas y el sistema mexicano de salud*
}

\author{
Pilar A. Parra**
}

El sistema de salud en México fue diseñado con base en el modelo occidental que enfatiza la medicina curativa en lugar de la preventiva. Sin embargo, la incorporación de las comadronas en los programas de atención materno-infantil y de planificación familiar de varias dependencias de salud pública es una excepción en las políticas gubernamentales de salud. Este artículo examina la importancia de las comadronas al analizar quiénes utilizan sus servicios entre las mujeres rurales. Esta investigación destaca que las mujeres analfabetas, pobres, con hijos, que viven en áreas remotas de difícil acceso confían en las comadronas. La necesidad que este sector de la población tiene de los servicios de las comadronas otorga mayor importancia a los programas gubernamentales, los cuales representan un caso único en el que ha ocurrido la incorporación de prácticas tradicionales a la concepción moderna de salud. Sin embargo, para poder instrumentar programas que tengan la capacidad de ofrecer a las mujeres rurales los beneficios de ambos sistemas de salud, la incorporación de las comadronas tradicionales requiere no sólo el mejoramiento de sus habilidades en las técnicas antisépticas modernas sino del reconocimiento de las aportaciones de las prácticas de salud tradicionales y de la investigación de los elementos y prácticas para impulsar su entendimiento.

\section{Introducción}

En este artículo se analizará brevemente la instrumentación de los programas de salud en las áreas rurales que no siguen el esquema curativo predominante en México, por lo cual se concentra en la

* Agradezco a Jorge Martínez Manautou, director de Planificación Familiar del Instituto Mexicano del Seguro Social (IMSS), el haberme permitido consultar la Encuesta Rural de Planificación Familiar 1981. También quiero expresar mi agradecimiento a Octavio Mojarro y al personal del Departamento de Estudios Demográficos del IMSS por su ayuda para documentar mi investigación sobre las comadronas. Agradezco asimismo a Doris P. Slesinger y a Max Pfiffer por sus valiosos comentarios y críticas y por su apoyo y estímulo durante el desarrollo de este artículo, asf como los comentarios de varios lectores que ayudaron a mejorarlo. versity.

** Institute for Health, Health Care Policy, and Aging Research, Rutgers Uni- 
incorporación de las comadronas tradicionales a los programas de salud materno-infantil y de planificación familiar. Primero presentaré, en forma breve, la incorporación de las comadronas al sistema institucional de salud. En segundo lugar, analizaré las características económicas, sociales y culturales de las mujeres rurales desagregadas por el tipo de prestador de servicios de salud que estuvo presente en el nacimiento del último hijo. Después evaluaré las determinantes del uso de comadronas y otros prestadores de salud entre las mujeres que viven en comunidades de menos de 2500 habitantes. Debe señalarse que las mujeres que sólo hablan lengua indígena fueron eliminadas de la encuesta en la que se basa este estudio (Encuesta Rural de Planificación Familiar, ERPF, IMSS, 1983). Por lo tanto, no se considera aquí la prestación del servicio de salud a la población indígena que no habla español.

La modernización del sistema de salud en México forma parte del plan de desarrollo más amplio que ha seguido el país en el siglo XX. Se construyó con base en el modelo occidental que enfatiza la medicina curativa sobre la preventiva. El énfasis en la medicina curativa, especialización y alta tecnología forma parte de la tendencia general de la medicina occidental de los países industriales avanzados en los que los estándares de vida y salud han mejorado hasta tal punto que la medicina preventiva llega a ser secundaria. Éste no es ęl caso de México, en donde prevalecen condiciones pobres para la mitad de la población y la medicina preventiva se perfila más adecuada con la realidad y las necesidades del país. El derecho a la salud y la responsabilidad estatal de atender las necesidades de salud de la población fueron establecidas en la Constitución de 1917. Desde 1920 el Estado mexicano ha tenido una participación activa en la organización y control del sistema de salud, así como en la formación de médicos y en la investigación médica. Sin embargo, el acceso a los servicios de salud está altamente estratificado para diferentes sectores de la sociedad, y la calidad de los servicios varía enormemente. Por lo tanto, la cobertura de los sistemas de salud en México es limitada, tanto espacial como socialmente (López Acuña, 1980). En las áreas rurales es donde el sistema de salud enfrenta su mayor reto. Ahí, los niveles de mortalidad, morbilidad y desnutrición reflejan la desigual distribución de la riqueza y la falta de acceso a los servicios.

La problemática que enfrenta la salud pública en México en los noventa tiene orf́genes muy complejos y son múltiples los problemas que aquejan al sistema de salud. Por ejemplo, el modelo occidental médico es muy costoso, desde la formación del personal hasta la infraestructura hospitalaria necesaria que requiere este modelo médico para su funcionamiento. Este problema se 
agudizó en las décadas de los setenta y los ochenta. La crisis económica que enfrentó el país en esos años afectó la capacidad del Estado para financiar los gastos del bienestar social. Durante esos años la tasa de inflación borró cualquier incremento en los gastos destinados a los servicios de salud, y después de 1982 el nivel de gasto real disminuyó. Más aún, personal médico, centros de salud, hospitales, y clínicas están concentrados en áreas urbanas, lo que impide la procuración rutinaria de servicios médicos a las áreas rurales. Si se establecen programas en sitios rurales, frecuentemente carecen de personal, no están suficientemente acondicionados y algunas veces existe duplicación en los servicios. La falta de continuidad de los programas establecidos por las administraciones gubernamentales sucesivas constituye también otro obstáculo para el desarrollo de un sistema nacional de salud efectivo para todos los sectores.

Actualmente, las instituciones de salud en México pueden agruparse en tres grandes sectores: 1) organizaciones públicas de seguridad social, entre las que destacan el Instituto Mexicano del Seguro Social (IMSS), que atiende a personas empleadas en dependencias no gubernamentales, y el Instituto de Seguridad y Servicios Sociales para los Trabajadores del Estado (ISSSTE), que atiende a los empleados del gobierno; 2) servicios para la población abierta, especialmente los que presta la Secretaría de Salud [SSA] que atiende a la mayoría de la población que no está contemplada en las dos organizaciones anteriormente mencionadas, y 3) la medicina privada, que incluye los servicios proporcionados por organizaciones religiosas. Mediante sus programas de solidaridad social, el IMSS-Coplamar y la SSA son los dos principales organismos que atienden a la población rural (UNAM, 1984).

Debido al desarrollo económico desigual de México, las causas más frecuentes de mortalidad en los sectores marginales de las áreas urbanas y entre la población rural continúan siendo la desnutrición y las enfermedades infecciosas y parasitarias. Por lo tanto, la naturaleza de la deficiente salud en México subraya la necesidad de establecer programas de salud comunitaria con medicina preventiva, como el mejoramiento del suministro de agua y drenaje, entrenamiento en higiene y sanitario, y el mejoramiento de los programas de inmunización (Ward, 1985: 129). Debe añadirse también que otros factores estructurales profundos subyacen en los problemas de salud en México. La mala situación económica general de la población rural debe mejorarse a fin de asegurar, entre otras cosas, un consumo nutricional más alto. La erradicación total y permanente de estos problemas va más allá de los parámetros de cualquier sistema de salud, y se requeriría de un 
cambio estructural radical para resolver el problema por completo.

Las políticas gubernamentales orientadas hacia la instrumentación de la salud enfatizan la medicina curativa en lugar de la preventiva (Stebbins, 1986). Sin embargo, han existido unos cuantos intentos, especialmente en las áreas rurales, para construir un puente entre la medicina tradicional y la moderna mediante la incorporación de elementos autóctonos de las comunidades a los programas de salud preventiva (Leñero, 1982). Ése es el caso de los programas de salud rural instrumentados por la SSA de 1976 a 1982 (Correu, 1980) y de la capacitación de comadronas autóctonas por diversas instituciones de salud pública, principalmente, la SSA, el IMSS y, a partir de 1972, el Instituto Nacional Indigenista (INI).

\section{Los programas de las comadronas}

En la década de los setenta, el IMSS y la SSA iniciaron un proyecto nacional para incorporar a las comadronas tradicionales al sistema institucionalizado de atención materno-infantil. Ello se hizo para mejorar la labor de las comadronas en cuestiones de salud mediante el establecimiento de un vínculo entre los servicios modernos y estas prestadoras tradicionales de salud. El objetivo de este esfuerzo era incrementar los conocimientos de las comadronas para que pudieran saber cuándo remitir a las mujeres o niños al puesto de salud más cercano y para ayudar a promover entre la población medidas de higiene que pudiesen facilitar las campañas de vacunación. Más tarde, el INI fue incorporado al programa para entrenar a las comadronas en los lugares donde habitan poblaciones que no hablan español (Zetina, 1979).

La capacitación de las comadronas ha enfrentado varios problemas durante el proceso, pero los resultados del programa también muestran evidencia de su éxito. Primero, debemos mencionar la continuidad del programa desde 1974. Han pasado tres periodos presidenciales y el programa permanece, algo inusual en el quehacer político mexicano. En general, se cree que los funcionarios de salud se dieron cuenta de que las comadronas no sólo tienen un importante papel en el cuidado de la salud maternoinfantil, sino que también significan un potencial como agentes en la planificación familiar. Por lo tanto, en 1977 tanto el IMSS como la SSA incluyeron en sus programas de capacitación para las comadronas una sección sobre planificación familiar. La política subyacente en estos programas, posteriores a 1977, refleja el interés por parte del gobierno de promover la planificación fami- 
liar en las áreas rurales (Martínez Manautou, 1986). Actualmente, la principal institución responsable del programa que reclutó y/o retuvo a las comadronas es el IMSS, que también es una de las instituciones más poderosas en la instrumentación de la planificación familiar en México.

Los resultados de la inclusión de las comadronas en el esfuerzo de planificación familiar han sido positivos. Las comadronas han tenido éxito en incorporar nuevas usuarias a la planificación familiar. Ha habido un incremento en el uso de anticonceptivos en las áreas rurales, de $5 \%$ en 1969 a $27 \%$ en 1981 . 6\% o 519664 de estas mujeres rurales fueron incorporadas a la planificación familiar por las comadronas (Correu et al., 1986). Los resultados positivos, que incluyen remitir los embarazos difíciles a los puestos de salud y el reclutamiento de mujeres al uso de anticonceptivos, han asegurado la sobrevivencia de los programas de las comadronas.

En el México rural, al mismo tiempo que se mejora la disponibilidad del servicio institucional de salud, persiste la confianza continuada en las comadronas. El mejoramiento del acceso a los servicios de salud públicos y privados durante los últimos 20 años ha tenido sólo un impacto modesto sobre la elección de las mujeres acerca de quién las auxiliará en el parto. $65 \%$ de todos los nacimientos en las áreas rurales fueron atendidos por comadronas tradicionales en 1969; para 1980 más de la mitad de los nacimientos aún se realizaron bajo la supervisión de una comadrona. Las principales razones que las mujeres oponen a los métodos modernos se relacionan con factores sociales, económicos y culturales. A las mujeres les disgusta ser alejadas de sus casas e hijos y dar a luz sin el apoyo de otros miembros de su familia (Cosminsky, 1977). También resienten la separación del recién nacido y el trato distante que reciben de las enfermeras y de los médicos (Jordan, 1980). Las dificultades económicas y de transporte son también otras razones importantes para rechazar el cuidado médico moderno (Young, 1981). Enseguida examinaré las características de las mujeres que utilizan a la comadrona para la atención maternoinfantil.

\section{Metodología}

Fuente de datos

El análisis se basa en la ERPF, una muestra nacional probabilística de hogares de mujeres entre los 15 y los 49 años de edad en localidades con menos de 2500 habitantes enumeradas en el censo de 
1970. Comprende 86 municipios que contienen 422 localidades, estratificadas según la disponibilidad de servicios del IMSS. Desafortunadamente, sólo fueron entrevistados los hogares en donde se hablaba español, con lo cual se eliminó a los hablantes de lengua indígena. Los instrumentos de la encuesta consistían en 1) un cuestionario de hogar, 2) un cuestionario individual para todas las mujeres en edad reproductiva enumeradas en el hogar y 3) un cuestionario que recababa información sobre cada comunidad comprendida en la muestra. Las entrevistas individuales fueron concluidas en 1981, habiéndose entrevistado a 8103 mujeres en edad reproductiva (IMSS, 1983). La información incluye datos sociodemográficos y una amplia gama de información sobre atención materno-infantil, embarazos e historia anticonceptiva.

Se seleccionó una submuestra de 4265 mujeres de las 8103 mujeres entrevistadas. Estas mujeres eran aquellas cuyo último periodo de embarazo había terminado entre 1976 y la fecha de la entrevista; se excluyeron los embarazos que terminaron en abortos. Para el análisis multivariado se excluyeron también 70 casos adicionales, debido a que la información sobre la comunidad y datos del hogar era incompleta.

\section{Descripción de las variables}

Seleccionamos las variables que proporcionaran información acerca de 1) características demográficas de las mujeres (edad, número de hijos y escolaridad); 2) nivel económico de su hogar medido a través de la actividad económica del jefe del hogar (i.e., la persona identificada como el principal aportante económico del hogar) (Zúniga et al., 1986) y la calidad de su vivienda (piso de cemento o de otros materiales), y 3) características de la localidad donde vivían. Teníamos particular interés en el grado de accesibilidad al mercado más cercano de la localidad, lo que expondría a las mujeres a otros servicios de salud o educativos, facilidades y prácticas no disponibles en sus pueblos. También se consideró el tamaño de la comunidad, bajo el supuesto de que las mujeres que vivían en pueblos más pequeños tendrían valores tradicionales más arraigados que aquellas que vivían en comunidades más grandes. Finalmente, la localización y el tipo de prestador de servicios de salud en el nacimiento del último hijo se codificó en cuatro categorías: "público" si fue atendido por personal con capacitación formal en una institución pública; "privado" si se encontraban presentes un médico privado o una enfermera; "comadrona" si el bebé nació con la ayuda de una comadrona tradi- 
cional, y "otros" si la mujer dio a luz a su hijo sola o sin la presencia de alguno de los practicantes mencionados anteriormente. Todas las variables y sus definiciones se muestran en el cuadro 1.

\section{Método analítico}

La primera parte del análisis consiste en comparar las características de las mujeres que seleccionaron alguno de los cuatro tipos de auxiliares para el parto. Los cuadros 2 y 3 presentan estos datos. El cuadro 4 presenta el análisis multivariado con variables seleccionadas del tipo de prestador de salud dentro de un modelo de regresión logística lineal. Se ajustó un modelo para predecir cada tipo de prestador de salud.

\section{Resultados}

\section{Las usuarias de comadronas}

Para describir las características de las usuarias de comadronas y otros prestadores de salud, examinamos las características demográficas y socioeconómicas de las mujeres así como del lugar de residencia.

El cuadro 2 resume las características demográficas de las mujeres de acuerdo con su elección de prestador de servicios de salud durante el parto.

La edad de las mujeres no parece estar relacionada con su elección de quién las atenderá durante el parto. Aproximadamente $57 \%$ de todas las mujeres confían en las comadronas para dar a luz. En general, la proporción de mujeres que prefieren a las comadronas se incrementa ligeramente entre el grupo de mayor edad. Sólo el 11\% de todas las mujeres de cada grupo de edad utiliza los servicios privados. La utilización de servicios públicos es mayor entre las mujeres de 20 a 34 años y disminuye después entre las mujeres de 35 años y más. Ello puede ser resultado de la confianza de las mujeres en su experiencia en los partos anteriores [Sokoloff, 1986]. Es importante señalar también que $8 \%$ de las mujeres no cuentan con los cuidados de algún asistente con capacitación formal o informal.

Hay una relación positiva entre el número de hijos y la confianza en el auxilio de las parteras tradicionales. La proporción de mujeres que prefieren los servicios de una comadrona durante el parto se incrementa con el número de hijos (de 51 a $60 \%$ ) y, por 
CUADRO 1

Variables categóricas, definición y porcentaje

\begin{tabular}{|c|c|c|}
\hline Variables categóricas & Definición & $\%$ \\
\hline Auxiliar en el parto & $\begin{array}{l}1 \text { Público: IMSS, IMSS-Coplamar, } \\
\text { ISSSTE, SSA } \\
2 \text { Privado: médicos, enfermeras } \\
3 \text { Comadrona: sólo la comadro- } \\
\text { na tradicional } \\
4 \text { Otros: curandero, trabajadora } \\
\text { social comunitaria, familia, sola }\end{array}$ & $\begin{array}{r}23.9 \\
11.5 \\
56.7 \\
8.0\end{array}$ \\
\hline Edad de la mujer & $\begin{array}{ll}1 & 15-19 \text { años } \\
2 & 20-34 \text { años } \\
3 & 35-49 \text { años }\end{array}$ & $\begin{array}{r}8.5 \\
64.5 \\
27.0\end{array}$ \\
\hline Número de hijos & $\begin{array}{ll}1 & 1-2 \text { hijos } \\
2 & 4-5 \text { hijos } \\
3 & 5 \text { y más hijos }\end{array}$ & $\begin{array}{l}27.6 \\
25.2 \\
47.2\end{array}$ \\
\hline Escolaridad de la mujer & $\begin{array}{l}1 \text { Sin instrucción } \\
2 \text { 1-3 años de primaria } \\
3 \text { 4-5 años de primaria } \\
46 \text { y más años de primaria u } \\
\text { otro nivel de escolaridad }\end{array}$ & $\begin{array}{l}24.8 \\
42.5 \\
16.6\end{array}$ \\
\hline
\end{tabular}

Actividad económica del jefe del hogar

No agrícola

1 Por su cuenta

2 Trabajador asalariado

Agrícola

1 Por su cuenta

2 Trabajador asalariado

Tipo de piso en la vivienda

1 Tierra

2 Cemento, madera, otros

Número de habitantes en la

1 Menos de 500

Accesibilidad al centro

1 Buena: carretera pavimentada y menos de 30 minutos

2 Regular: carretera de terracería y menos de una hora

3 Mala: pavimentada o de terracería y más de una hora

Fuente: Encuesta Rural de Planificación Familiar. ERPF, 1981.

el contrario, el grupo de mujeres que escoge los servicios públicos o privados tiende a disminuir a medida que tiene más hijos.

La escolaridad parece ser el factor más importante que reduce 


\section{CUADRO 2}

Características demográficas de las mujeres con un embarazo a término por tipo de auxiliar de salud en el parto

\begin{tabular}{|c|c|c|c|c|c|}
\hline \multirow{2}{*}{$\begin{array}{l}\text { Características } \\
\text { de Ia mujer }\end{array}$} & \multicolumn{4}{|c|}{ Auxiliar de salud en el parto $(\%)$} & \multirow{2}{*}{$\begin{array}{c}\text { Total } \\
\text { (núm.) }\end{array}$} \\
\hline & Público & Privado & Comadrona & Otros & \\
\hline \multicolumn{6}{|l|}{ Grupo de edad } \\
\hline $15-19$ & 23.5 & 11.6 & 57.4 & 7.5 & 362 \\
\hline $20-34$ & 24.7 & 11.2 & 56.0 & 8.1 & 2750 \\
\hline $35-49$ & 21.4 & 11.8 & 58.4 & 8.4 & 1145 \\
\hline Total & 23.7 & 11.4 & 56.7 & 8.3 & 4257 \\
\hline \multicolumn{6}{|l|}{ Número de hijos } \\
\hline $1-2$ & 28.4 & 14.9 & 50.8 & 5.9 & 1182 \\
\hline $3-4$ & 24.2 & 10.2 & 57.5 & 8.1 & 1069 \\
\hline $5+$ & 20.7 & 10.0 & 59.8 & 9.5 & 2006 \\
\hline Total & 23.7 & 11.4 & 56.7 & 8.2 & 4257 \\
\hline \multicolumn{6}{|l|}{ Escolaridad } \\
\hline Sin instrucción & 14.7 & 7.4 & 65.5 & 12.4 & 1061 \\
\hline Primaria: (1-3 años) & 22.4 & 9.5 & 59.4 & 8.6 & 1807 \\
\hline Primaria: (4-5 años) & 24.9 & 14.0 & 55.6 & 5.6 & 706 \\
\hline \multicolumn{6}{|l|}{6 y más años } \\
\hline completos & 39.9 & 20.2 & 37.1 & 2.8 & 678 \\
\hline Total & 23.7 & 11.4 & 56.7 & 8.1 & 4252 \\
\hline
\end{tabular}

la presencia de una comadrona durante el parto, entre las mujeres rurales. Así, $66 \%$ de las mujeres sin escolaridad fueron atendidas por una comadrona en su último parto y el $15 \%$ por un servidor público. Por el contrario, pocas mujeres con escuela primaria completa acudieron a una comadrona ( $37 \%$ ) y más mujeres asistieron a un servicio público (40\%). También se incrementó el uso de servicios privados de salud entre las mujeres con mayor nivel de escolaridad. $20 \%$ de las mujeres con seis o más años de escuela utilizaron los servicios privados en su último parto. Frecuentemente se ha interpretado que el nivel de escolaridad es el factor más importante que aporta valores "modernos" y cambia los patrones culturales tradicionales de conducta entre la población rural; estos datos apoyan ese enfoque. Sin embargo, el nivel de escolaridad puede estar relacionado también con mayores ingresos, lo que permite la elección de prestadores de servicios de salud alternativos.

Para resumir, casi la misma proporción de mujeres rurales de todas las edades tuvo una comadrona como su asistente durante el parto, con sólo una ligera tendencia hacia los servicios de una comadrona en las edades mayores. Las mujeres de más edad, con 
varios embarazos previos y menor nivel de escolaridad, son atendidas en general por las parteras tradicionales. La escolaridad de las mujeres está claramente relacionada con la elección de un prestador de servicios de salud en el momento del parto. Un mayor número de años de escolaridad también está asociado con una mejor situación económica. Ambos factores afectan la demanda de los servicios públicos y privados de salud.

También es importante considerar cómo se relacionan las diferencias del bienestar económico de los hogares y las características de la comunidad con la elección del prestador de servicios de salud. El cuadro 3 resume el tipo de actividad económica del

CUADRO 3

Características del hogar y de la comunidad de las mujeres

con un embarazo a término por tipo de auxiliar de salud en el momento del parto

\begin{tabular}{|c|c|c|c|c|c|}
\hline \multirow{2}{*}{$\begin{array}{l}\text { Características } \\
\text { del hogar y de } \\
\text { la comunidad }\end{array}$} & \multicolumn{4}{|c|}{ Auxiliar de salud en el parto (\%) } & \multirow{2}{*}{$\begin{array}{l}\text { Total } \\
\text { (núm. }\end{array}$} \\
\hline & Público & Privado & Comadrona & Otros & \\
\hline \multicolumn{6}{|l|}{$\begin{array}{l}\text { Actividad económica del } \\
\text { jefe del hogar no agrícola }\end{array}$} \\
\hline $\begin{array}{l}\text { Por su cuenta } \\
\text { Trabajador asalariado }\end{array}$ & 24.2 & 17.6 & 51.5 & 6.7 & 420 \\
\hline agrícola & 36.9 & 12.9 & 43.9 & 6.3 & 1000 \\
\hline Por su cuenta & 16.0 & 9.4 & 64.5 & 10.1 & 2027 \\
\hline Trabajador asalariado & 26.1 & 9.9 & 57.7 & 6.3 & 660 \\
\hline Total & 23.5 & 11.2 & 57.1 & 8.2 & 4107 \\
\hline \multicolumn{6}{|l|}{$\begin{array}{l}\text { Condiciones de la vivienda } \\
\text { Tipo de piso }\end{array}$} \\
\hline De tierra & 17.6 & 7.8 & 64.1 & 10.5 & 2226 \\
\hline Cemento, madera, otros & 30.4 & 15.3 & 48.7 & 5.6 & 2030 \\
\hline Total & 23.7 & 11.4 & 56.7 & $8: 2$ & 4256 \\
\hline \multicolumn{6}{|l|}{ Núm. de habitantes } \\
\hline Menos de 500 & 22.4 & 10.8 & 58.0 & 8.8 & 2226 \\
\hline $500-999$ & 22.1 & 9.5 & 58.9 & 9.6 & 1057 \\
\hline $1000-2499$ & 27.7 & 14.2 & 52.4 & 5.6 & 1204 \\
\hline Total & 23.8 & 11.4 & 56.6 & 8.1 & 4223 \\
\hline \multicolumn{6}{|c|}{ Accesibilidad al centro comercial } \\
\hline Buena & 38.0 & 14.2 & 41.5 & 6.3 & 1167 \\
\hline Regular & 19.3 & 14.5 & 60.2 & 6.0 & 1186 \\
\hline Mala & 17.6 & 7.8 & 64.0 & 10.6 & 1904 \\
\hline Total & 23.7 & 11.4 & 56.7 & 8.2 & 4257 \\
\hline
\end{tabular}

Fuente: Encuesta Rural de Planificación Familiar, ERPF, 1981. 
jefe del hogar, las condiciones materiales de la vivienda, el tamaño de la comunidad y la facilidad de acceso al centro comercial más cercano. En la encuesta, se definió al jefe de familia como la persona que contribuye con los mayores ingresos económicos de la familia. La actividad económica principal se clasificó como agrícola y no agrícola, y se desglosó en si el jefe del hogar era trabajador por su cuenta o trabajador asalariado (Zúñiga et al., 1986).

Las mujeres cuyos esposos son trabajadores asalariados en una actividad no agrícola tienden a utilizar los servicios públicos de salud en lugar de los servicios de las parteras tradicionales. Esto puede deberse al hecho de que los trabajadores asalariados en actividades no agrícolas tienen mayor probabilidad de estar asegurados por su patrón y de recibir los beneficios de salud del IMSS o del ISSSTE, lo cual contrasta con los trabajadores agrícolas asalariados quienes, en general, no tienen acceso al seguro de salud. Esta situación se refleja entre las mujeres cuyos esposos son trabajadores asalariados en la agricultura. $58 \%$ de estas mujeres fueron atendidas en el momento del parto por una comadrona. Las esposas de los trabajadores por su cuenta en actividades no agrícolas (52\%) tienden a confiar menos en las comadronas que las esposas de los trabajadores por su cuenta en la agricultura [65\%). Este grupo tiene la mayor proporción de mujeres atendidas por una comadrona entre todos los grupos ocupacionales. También es importante señalar que $10 \%$ de las mujeres en la misma categoría fueron atendidas por algún familiar, un curandero o estuvieron solas, resultados que no son de sorprender dado que los trabajadores por su cuenta en la agricultura se encuentran entre los grupos ocupacionales más pobres debido a la pequeña cantidad de tierra que la mayoría de ellos posee. ${ }^{1}$ A los trabajadores agrícolas asalariados cuyos salarios son menores que los de los empleados de oficina no les va mucho mejor. Una proporción ma-

\footnotetext{
${ }^{1}$ Zúñiga et al. reportan (pp. 185-187) que en el México rural 76\% de los propietarios de tierra tiene menos de cuatro hectáreas, otro $17 \%$ tiene entre cinco y doce hectáreas, y $7 \%$ posee 13 hectáreas o más. Por lo tanto, la mayoría (93\%) de los propietarios cuyo principal ingreso está basado en la producción agricola pueden ser considerados en general como los menos afortunados comparados con los propietariós que están involucrados en actividades económicas no agrícolas. Actualmente, entre los campesinos del México rural la propiedad de la tierra no necesariamente es equivalente al bienestar, debido a que se requieren ocho hectáreas de tierra de temporal para la sobrevivencia de una familia promedio de 5.5 personas. Para reproducir la unidad familiar de una familia promedio de 5.5 personas, más allá de los límites de la mera subsistencia, se requieren 12 hectáreas. Por lo tanto, sólo $7 \%$ de las familias campesinas que se consideran trabajadorés por su cuenta en la agricultura pueden generar excedentes de la producción agrícola (IMSS, 1983).
} 
yor de las mujeres que pertenecen a estos grupos es auxiliada por comadronas, y otra proporción menor asiste a los servicios públicos o privados.

Otros indicadores de la situación económica de las mujeres rurales y de sus hogares son las condiciones materiales de la vivienda. Consideramos los materiales de construcción de los pisos como otro indicador económico. Más de $60 \%$ de las mujeres que habitaban viviendas de piso de tierra eran atendidas por una comadrona y sólo una cuarta parte fue auxiliada por un prestador de servicios de salud, público o privado.

El tamaño del lugar de residencia es otro factor que afecta el tipo de auxiliar en el momento del parto. Una proporción mayor de mujeres que viven en poblados con menos de 1000 habitantes utilizan los servicios de una comadrona que las mujeres que viven en comunidades más grandes. En lugares con 1000 a 2499 habitantes, la proporción de mujeres que asisten a los servicios públicos de salud se incrementa de 22 a $28 \%$, y la proporción de mujeres que acuden a los servicios privados de 11 a $14 \%$, lo cual puede ser resultado de factores culturales. Los valores tradicionales se observan con mayor fuerza en las comunidades pequeñas, donde resulta más difícil la aceptación de la medicina moderna. También puede ser resultado de la falta de servicios de salud alternativos accesibles, problemas de transporte, o ambos. La accesibilidad a otras comunidades se mide por el tipo de carretera y tiempo de traslado al pueblo más cercano con el que la gente de la comunidad comercia más frecuentemente. En general, estas localidades son comunidades más grandes en donde existen diversos servicios, tales como centros de salud, bancos y oficinas locales de gobierno, que no están disponibles en sus villorrios. $38 \%$ de las mujeres que vivían en comunidades en las que una carretera pavimentada o de terracería conducía a una localidad mas grande, y el tiempo de traslado era menor a 30 minutos, escogieron un servicio público de salud para dar a luz a su último hijo, y $14 \%$ eligieron un servicio privado de salud cuando el acceso era bueno o regular. La clientela de las comadronas se redujo a $42 \%$ cuando existían buenas condiciones de acceso a la localidad comercial más cercana. Por el contrario, cuando el acceso a la localidad comercial requería más de una hora, una proporción más reducida se atendió con un servicio de salud público o privado $(18$ y $8 \%$, respectivamente) y $64 \%$ acudió a una comadrona.

En resumen, las mujeres que viven en hogares en los que la actividad económica principal es la agricultura y que habitan viviendas muy modestas representan los estratos más pobres del México rural. El principal prestador de servicios de salud para 
estas mujeres es la partera tradicional. También las mujeres que viven en las comunidades más pequeñas, con comunicación deficiente y alejadas de un centro comercial, favorecen a las comadronas.

Debido a que muchas de estas características individuales están interrelacionadas, es difícil identificar los factores que influyen en la utilización del prestador de servicios de salud durante el parto. Es preciso realizar un análisis multivariado de las variables individuales, económicas y de la comunidad para permitir la medición de la fuerza relativa y la contribución que aporta cada uno de los factores.

\section{Análisis multivariado}

Debido a que la variable dependiente es categórica, se eliminó como posible procedimiento analítico el método común de los minimos cuadrados y se prefirió utilizar el análisis de regresión logística (Hanushek y Jackson, 1977). Se ajustaron cuatro modelos para probar los factores que afectan la elección del servicio público, privado o de una comadrona u otro tipo de auxiliar en el momento del nacimiento del último bebé. Cada uno de los cuatro modelos representa un evento dicotómico, definido como un auxiliar de salud versus todas las demás posibilidades. Los resultados se presentan en el cuadro 4.

Como no era posible observar las probabilidades, y los efectos sobre los logitos son más difíciles de interpretar, se exponenciaron los coeficientes de la regresión logística para que resultaran fracciones impares que permitieran la interpretación como efectos de los impares en lugar de los logitos. Las fracciones impares miden cuánto se incrementa o se reduce la probabilidad de una categoría a otra. Si el coeficiente logito estimado es negativo, el valor exponencial será menor a uno. Si es positivo, el valor exponencial será mayor a uno.

\section{Resultados}

El análisis muestra que los factores sociales, culturales y económicos tienen un efecto fuerte sobre la selección de la comadrona. $\mathrm{Si}$ se observa primero el modelo 3 en el cuadro 4 , el predictor más fuerte para la selección de una comadrona es la falta de acceso a la localidad comercial más cercana: Después de controlar los factores individuales y económicos, las mujeres que viven en un po- 


\section{CUADRO 4}

Coeficientes de regresión logística, errores estándar y fracciones impares para modelos logitos que estiman el tipo de auxiliar de salud durante el parto

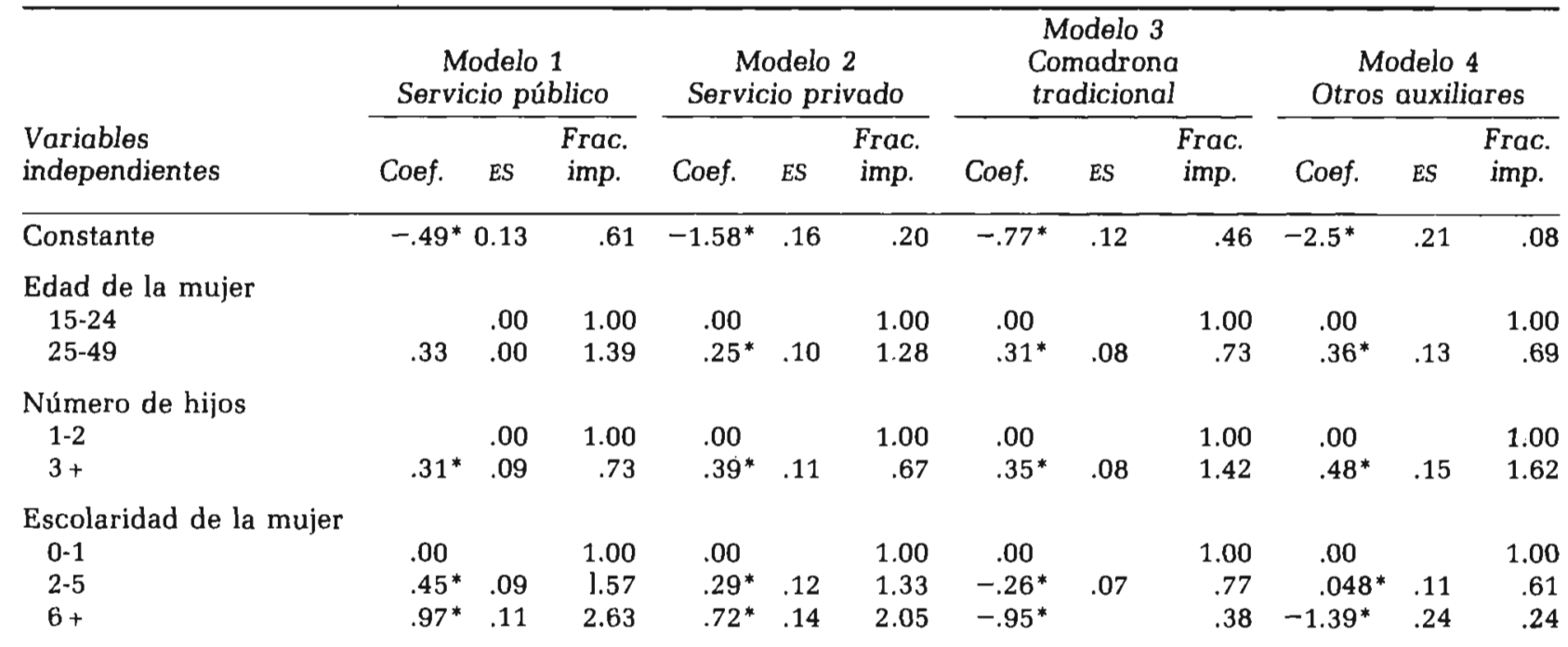




\begin{tabular}{|c|c|c|c|c|c|c|c|c|c|c|c|c|}
\hline \multicolumn{13}{|l|}{ Tipo de vivienda } \\
\hline Cemento/otros & .00 & & 1.00 & .00 & & 1.00 & .00 & & 1.00 & .00 & & 1.00 \\
\hline Tierra & $-.39^{*}$ & .07 & .67 & $-.52^{*}$ & .09 & .59 & $.45^{*}$ & .06 & 1.56 & $.48^{*}$ & .11 & 1.61 \\
\hline \multicolumn{13}{|c|}{ Tamaño de la comunidad } \\
\hline Menos de 500 & .00 & & 1.00 & .00 & . & 1.00 & .00 & & 1.00 & .00 & & 1.00 \\
\hline $500-999$ & $-.21 *$ & .08 & .81 & $-.25^{*}$ & .11 & .77 & $.23^{*}$ & .08 & 1.25 & $.26^{*}$ & .13 & 1.29 \\
\hline $1000-2499$ & .11 & .08 & .90 & .00 & .10 & .00 & .10 & .07 & .90 & -.23 & .14 & .79 \\
\hline \multicolumn{13}{|c|}{$\begin{array}{l}\text { Carretera y tiempo al centro } \\
\text { comercial más cercano }\end{array}$} \\
\hline Bueno & .00 & & 1.00 & .00 & & 1.00 & .00 & & 1.00 & .00 & & 1.00 \\
\hline Regular & $-.85^{*}$ & .09 & .42 & $.27^{*}$ & .10 & 1.30 & $.68^{*}$ & .08 & 1.97 & .12 & .16 & .88 \\
\hline Malo & $-.82^{*}$ & .08 & .44 & $-.34^{*}$ & .10 & .71 & $.77^{*}$ & .08 & 2.16 & $.46^{*}$ & .14 & 1.58 \\
\hline $\mathrm{N}$ & \multicolumn{3}{|c|}{4195} & \multicolumn{3}{|c|}{4195} & \multicolumn{2}{|c|}{4195} & & \multicolumn{3}{|c|}{4195} \\
\hline \multicolumn{13}{|c|}{$\mathrm{X}^{2}$ para falta de efecto } \\
\hline de las variables & \multicolumn{3}{|c|}{323.2} & \multicolumn{3}{|c|}{160} & \multicolumn{2}{|r|}{349.1} & & \multicolumn{3}{|c|}{142.3} \\
\hline D.F. & \multicolumn{3}{|c|}{9} & \multicolumn{3}{|c|}{9} & \multicolumn{2}{|r|}{9} & & \multicolumn{3}{|c|}{9} \\
\hline
\end{tabular}

- Significativas al nivel .05. Un coeficiente es significativo si su valor es al menos 1.96 de su error estándar.

Fuente: Encuesta Rural de Planificación Familiar, ERPF, 1981. 
blado en el que se requiere más de media hora de traslado por carretera sin pavimentar para llegar a la localidad comercial más cercana, las fracciones impares estimadas de recurrir a una comadrona se incrementan en $\mathbf{9 7 \%}$, comparadas con el grupo de mujeres que tiene acceso a una carretera pavimentada y que requiere menos de media hora para su traslado. Las fracciones impares más que se duplican cuando las mujeres vivían a más de una hora de distancia de la población con que comercian. Sin embargo, no hay un cambio significativo acerca de utilizar una comadrona en términos del tamaño de la comunidad. Este hecho refuerza la importancia de la falta de acceso a una comunidad más grande en la selección de una comadrona.

El segundo efecto más fuerte que favorece la utilización de una comadrona fue, como se esperaba, el factor económico medido por el tipo de piso de la vivienda. Si se controlan todos los demás factores, las mujeres que viven en viviendas con piso de tierra representaban un incremento de $56 \%$ en las fracciones impares de acudir a una comadrona, comparadas con el grupo de mujeres que habitaban viviendas con piso de cemento o de otros materiales.

Como se observó anteriormente, a medida que las mujeres tienen mayor escolaridad, menor es la probabilidad de elegir un auxiliar tradicional en el parto. El análisis demuestra que las fracciones impares relativas de ser atendida por una comadrona disminuyeron con cada categoría de escolaridad. Las mujeres con dos a cinco años de escolaridad tienen $23 \%$ menos probabilidad de acudir a una comadrona que las mujeres sin escolaridad o con un año de escolaridad. Las mujeres con seis o más años de escolaridad tienen $62 \%$ menos probabilidades de recurrir a una comadrona. La escolaridad se asocia con un mejoramiento en el bienestar del hogar y como auxiliar en el cambio de actitudes hacia prácticas nuevas. Por lo tanto, sugeriria que los factores económicos, sociales y culturales se ven reforzados por la asociación inversa entre la escolaridad de la mujer y la selección de una comadrona.

Finalmente, se esperaba que las mujeres de mayor edad con mayor número de hijos preferirían una comadrona debido a la confianza desarrollada a través de las experiencias previas en el proceso de labor de los partos. Sin embargo, los resultados indican que las mujeres de mayor edad - después de controlar todos los otros efectos- no favorecen a la comadrona sino que prefieren los servicios de salud públicos o privados. Potter sugiere que las mujeres de más edad escogen los servicios públicos porque pueden recibir sin cargo la ligadura de trompas inmediatamente después del parto si éste se realiza en un hospital público (Potter, 
1988), lo cual forma parte de la política gubernamental de planificación familiar (Alarcón, 1986). Sin embargo, como se mostró en el cuadro 2, el número de hijos es también un predictor fuerte para la elección de una comadrona. Cuando se controlan todos los demás factores, incluyendo la edad de las mujeres, la paridad del parto (o las experiencias previas con el nacimiento) aún aparece como un determinante significativo que lleva a las mujeres a acudir a las comadronas.

Es interesante señalar que hay una inversión consistente de resultados entre la selección de una auxiliar tradicional en el parto (modelo 3) y la selección de la medicina formal (modelos 1 y 2). Los resultados muestran que, por una parte, las mujeres pobres que vivían en áreas lejanas de difícil acceso, analfabetas y con hijos, confiaban en las comadronas. Por otra parte, las mujeres que tuvieron su primer o segundo hijo, mujeres de mayor edad, con mayor nivel de escolaridad y con mejores condiciones económicas favorecieron a los servicios públicos o privados. También el acceso difícil a la localidad comercial más cercana está asociado negativamente con el uso de servicios públicos o privados. El modelo 4 dirige la atención al pequeño pero importante $10 \%$ de las mujeres que quedan fuera de los auxiliares formales o informales durante el parto. Este grupo se comporta en forma similar al grupo de mujeres del modelo 3; esto es, en general las mujeres que no contaron con algún auxiliar capacitado en su último parto son similares a las que escogieron a una comadrona. Tienden a ser pobres, analfabetas, y vivían en comunidades lejanas. Es importante mencionar que las mujeres de más edad tenían $31 \%$ menos probabilidad de estar solas, con un miembro de la familia o con un curandero en el parto de su último hijo que las mujeres más jóvenes, aun después de controlar la paridad. Ello sugiere que las mujeres de mayor edad tienen más conocimiento de los riesgos del parto y buscan el cuidado de las instituciones o de las comadronas.

\section{Conclusiones}

Este artf́culo estudia el fenómeno de la incorporación de las comadronas tradicionales al sistema institucional de salud que atienden a los sectores rurales del país. Se argumenta que la inclusión de las comadronas tradicionales es el resultado del fracaso del sistema público de salud en proporcionar la misma calidad en el cuidado de la salud en las áreas rurales similar al de las áreas urbanas, y de la instrumentación de las políticas de población.

Históricamente, el proceso de modernización y, particular- 
mente, el desarrollo del sistema de salud han conducido a un progreso desigual y sesgado de las áreas urbanas sobre las rurales. Proporcionar los servicios de salud a las áreas rurales ha sido difícil debido a la falta de infraestructura rural y a las barreras culturales. Además, el modelo occidental de salud favoreció a la medicina curativa sobre la preventiva y la tradicional y adoptó tecnologia y conocimientos extranjeros que devaluaron el conocimiento y las prácticas de salud nativas. Fue con la crisis económica, que se presentó al inicio de los años setenta, que surgieron las medidas de política gubernamental que vieron favorablemente a los practicantes tradicionales de la salud.

Én ese contexto, esta investigación analizó a las mujeres usuarias de los servicios de las comadronas. Los resultados de este análisis sostienen la noción de que las mujeres rurales prefirieron los servicios de una comadrona. Los datos demuestran que más de la mitad de todas las mujeres rurales confían en las comadronas (Mojarro y Cárdenas, 1986) y que factores sociales, culturales y económicos afectan fuertemente la selección de una comadrona. Además de su condición económica, las mujeres con más hijos, que vivían en comunidades más lejanas y con poco acceso a poblaciones más grandes, tenían más probabilidad de acudir a los servicios de una comadrona durante el nacimiento de su último hijo. Al mismo tiempo, la actual crisis económica del gobierno mexicano, exacerbada por su deuda con el Fondo Monetario Internacional y con otras instituciones internacionales, impide un mayor desarrollo del sistema de salud que cubriera a todas las comunidades rurales. A pesar de la instalación de más de 3000 puestos de salud del sistema IMSS-Coplamar a finales de los años setenta y principios de los ochenta, y de los esfuerzos de la SSA por expandir el número de hospitales rurales, es claro que muchas áreas lejanas no han sido alcanzadas por estos programas. No obstante, los datos demuestran que precisamente son las mujeres que viven en comunidades remotas las que usan los servicios de las comadronas.

Estos resultados tienen implicaciones políticas importantes. En primer término, debería otorgarse prioridad al desarrollo de programas que incorporen a las comadronas al sistema de salud en las áreas remotas con acceso limitado a las instituciones formales de cuidado de la salud. En segundo lugar, se requiere del mejoramiento del sistema de referencia para que las comadronas puedan canalizar los casos difíciles a los servicios formales de salud. Se sugieren también cambios tanto en el contenido de los programas para las comadronas - que, basados exclusivamente en la obstetricia de la medicina moderna, no incorporan los conoci- 
mientos que las comadronas han adquirido en sus largos años de práctica- como en los métodos de enseñanza, que requieren un alto nivel de escolarización del que más de la mitad de las comadronas en general carecen (Jordan, 1989). Cada vez es más evidente que los recursos autóctonos pueden contribuir a las estrategias autónomas de desarrollo y reducir la dependencia en las prácticas médicas costosas que predominan actualmente. La incorporación de las comadronas otorga a estas mujeres el reconocimiento al valioso servicio que han venido proporcionando en sus comunidades durante siglos y a la herencia cultural que representan, y que, hasta fecha reciente, fue considerada obsoleta, impráctica o primitiva.

Los programas que utilizan los servicios de las comadronas han estado vigentes por más de 15 años a escala nacional. Estos programas son un caso único en el que ha ocurrido la incorporación de la medicina tradicional al sistema de salud moderno. Hasta ahora el puente entre la medicina tradicional y la moderna ha seguido predominantemente una sola dirección, de lo moderno a lo tradicional. Sin embargo, para instrumentar programas que tengan la capacidad de ofrecer a las mujeres rurales los beneficios de ambos sistemas de salud, la incorporación de las comadronas tradicionales requiere no sólo del mejoramiento de sus habilidades con técnicas antisépticas modernas, sino también del reconocimiento de las contribuciones de las prácticas tradicionales de salud y de la investigación de sus elementos y prácticas para impulsar su conocimiento.

\section{Bibliografía}

Alarcón, N. y J. Martínez Manautou (1986). Innovaciones administrativas del Programa de Planificación Familiar, México.

Correu, Sergio (1980). Programa de salud rural, México, sSA.

Correu A.S., S.F. García y G.J. Cabello (1986). "Los servicios de planificación familiar en el medio rural", en J. Martínez Manautou (comp.), Planificación familiar, población y salud en el México rural, México, IMSS.

Cosminsky, Sheila (1977). "El papel de la comadrona en Mesoamérica", en América Indígena, vol. 37, núm. 2.

Hanushek, E. y J.E. Jackson (1977). Statistical Methods for Social Scientist, Nueva York, Academic Press.

IMSS (1983). Encuesta Rural de Planificación Familiar, 1981 (ERPF, 1981), México, Documento metodológico.

Jordan, Brigitte (1980). Birth in Four Cultures, Canadá, Eden Press. 
(1989). "Cosmopolitan Obstetrics: Some Insights from the Training of Traditional Midwives", en Soc. Sci. Med., vol 28, núm. 9.

Leñero, Elú de (1982). De lo institucional a lo comunitario, México, AMES. López Acuña, D. (1980). La salud desigual en México, México, Siglo XXI Editores.

Mojarro, O. y C. Cárdenas (1986). "Cobertura y disponibilidad de los servicios de atención materno-infantil", en J. Martínez Manautou (ed.), Planificación familiar, población y salud en el México rural, México, IMSS.

Potter E., Joseph (1988). "Utilización de los servicios de salud materna en el México rural", en Salud Pública Mexicana, vol. 30, núm. 3.

Sokoloff, Shoshana (1986). The Proud Midwives of Juchitan: Primary Health Providers in a Two Referral System, Harvard Medical School.

Stebbins, K. (1986), "Curative Medicine, Preventative Medicine and Health Status: The Influence of Politics on Health Status in a Rural Mexican Village", Soc. Sci. Med., vol. 23, núm. 2.

UNAM (1984). Hacia ù sistema nacional de salud 1883-1983, México.

Ward, Peter (1985). Welfare Politics in Mexico: Papering over the Cracks, Londres, Allen and Unwin.

Young, J.C. (1981). Medical Choice in a Mexican Village, Nueva Jersey, Rutgers University Press.

Zetina, Guadalupe (1979). Aceptabilidad e incorporación de la partera empírica a la prestación de servicios de planificación familiar, México, IMSS.

Zúñiga, E., D. Hernández, C. Menkes y C. Santos (1986). Trabajo familiar, conducta reproductiva y estratificación social, México, IMSS.

Zúñiga, E. et al. (1986). "Conducta reproductiva de los grupos sociales del área rural", en J. Martínez Manautou (ed.), Planificación familiar, población y salud en el México rural, México, iMSS. 